\title{
The hippocampus and spatial constraints on mental imagery
}

\author{
Chris M. Bird ${ }^{1,2}$, James A. Bisby ${ }^{2,3}$ and Neil Burgess ${ }^{2,3 *}$ \\ 'School of Psychology, University of Sussex, Brighton, UK \\ 2 Institute of Cognitive Neuroscience, University College London, London, UK \\ ${ }^{3}$ Institute of Neurology, University College London, London, UK
}

Edited by:

Joel Voss, Northwestern University Feinberg School of Medicine, USA

\section{Reviewed by:}

Joel Voss, Northwestern University Feinberg School of Medicine, USA Adam Johnson, Bethel University, USA

\section{${ }^{*}$ Correspondence:}

Neil Burgess, Institute of Cognitive Neuroscience, University College London, 17 Queen Square, London WC1N 3AR, UK.

e-mail: n.burgess@ucl.ac.uk
We review a model of imagery and memory retrieval based on allocentric spatial representation by place cells and boundary vector cells (BVCs) in the medial temporal lobe, and their translation into egocentric images in retrosplenial and parietal areas. In this model, the activity of place cells constrain the contents of imagery and retrieval to be coherent and consistent with the subject occupying a single location, while the activity of head-direction cells along Papez's circuit determine the viewpoint direction for which the egocentric image is generated. An extension of this model is discussed in which a role for grid cells in dynamic updating of representations (mental navigation) is included. We also discuss the extension of this model to implement a version of the dual representation theory of post-traumatic stress disorder (PTSD) in which PTSD arises from an imbalance between weak allocentric hippocampal-mediated contextual representations and strong affective/sensory representations. The implications of these models for behavioral, neuropsychological, and neuroimaging data in humans are explored.

Keywords: computational model, fMRI, place cell, boundary vector cell, construction

\section{INTRODUCTION}

Consider remembering an event such as a concert that you went to some time ago. At first you probably only retrieve a few bare facts about the event such as where it took place, who performed and roughly how long ago it took place. However, if asked, most people will be able to form a mental image of being at the concert, typically centered upon where they were standing or sitting, and from this vantage, visualize where the stage and other landmarks were located, and who else was nearby. The formation of this kind of mental image may well prompt the retrieval of other details that are incorporated into the imagined scene.

This kind of rich mental imagery for events in the past is considered a hallmark characteristic of episodic recollection (Tulving, 1985). Many researchers have argued that the hippocampus plays a critical role in this process (Kinsbourne and Wood, 1975; O'Keefe and Nadel, 1978; Moscovitch, 1995; Eichenbaum and Cohen, 2001). Rather more controversial is the suggestion that the hippocampus is critical for constructing a coherent mental image of a scene irrespective of whether that scene is a memory or newly invented (Becker and Burgess, 2001; Byrne et al., 2007; Hassabis et al., 2007b; Schacter et al., 2007), but see Squire et al. (2010). This is the position that we will advance in this review. In particular, we will describe the representations supported by the hippocampus that make such imagery possible. In keeping with the spirit of the articles in this series, we will argue that the role of the hippocampus in mental imagery is not necessarily tied to long-term memory.

Our position is inspired by over 30 years of research investigating the spatial firing properties of neurons in the hippocampus of rodents. The strength of this approach is that it allows us to specify what sort of information is represented at the level of neurons and to make predictions about how the nature of these representations will influence behavioral and neurophysiological measures. Of course, the model would not be valid if, (1) there was little correspondence between the function of the hippocampus in humans and rodents, or (2) humans use different brain regions for mental imagery of spatial scenes than they do for orienting and navigating within real environments. We will present data to address these issues. In the final section we shall detail how the model can explain the way in which imagery can go wrong in conditions such as post-traumatic stress disorder (PTSD).

In the concert example described above, mental imagery involves simulating being in a specific environment. Whether imagining past, future, or fictitious events, we typically try and retrieve topographical features to define a coherent space within which to locate ourselves, and then populate this space with objects consistent with that environment. Of course, the content of our mental images are based on our experiences, either wholly in the case of real memories, or partially, when imagining "future" or fictitious events based on our general knowledge of the world. It is the process of combining this knowledge into a dynamic, spatially coherent mental image that we argue requires the hippocampus.

\section{NEURAL REPRESENTATIONS OF SPACE IN RODENTS}

An obvious starting point for building a model of mental imagery for scenes is to try and understand how space is represented in the brain. A breakthrough in the understanding of this process occurred with the discovery of place cells (O'Keefe and Dostrovsky, 1971). Place cells recorded in freely moving rats 
each fire whenever the animal enters a specific portion of its environment (the "place field": O'Keefe, 1976). The firing of these cells is independent of the orientation of the rat during free exploration of open environments (Muller et al., 1994). Further experiments demonstrated that place cell firing correlates with behavioral responses in some spatial memory tasks (O'Keefe and Speakman, 1987; Lenck-Santini et al., 2001). We can, therefore, infer that the representation of location supported by place cells is behaviorally relevant to the animal.

To investigate the form of the sensory input to place cells, O'Keefe and Burgess (1996) varied systematically the shape and size of the rat's environment while recording from the same set of place cells. Distortions to the environments had a number of effects on the pattern of firing such that place fields stretched or became bimodal when the environment expanded. These patterns were consistent with place cell firing being a thresholded sum of inputs tuned to respond to the presence of a boundary at a given distance along a given allocentric direction (i.e., independent of the orientation of the rat). These hypothetical inputs were termed "boundary vector cells" (BVCs). By fitting a place cell's firing pattern across several different environmental shapes, the model could successfully predict its firing pattern in environments of novel shape (O'Keefe and Burgess, 1996; Hartley et al., 2000), see Figure 1A. More recently, the proposed BVCs, i.e., cells responding only to the distance and allocentric direction from the rat of an extended environmental boundary, were found in the subiculum (Lever et al., 2009), see Figure 1B. It also seems likely that "border cells" reported in the entorhinal cortex (Solstad et al., 2008) are a subset of a population of BVCs that happen to fire close to a boundary in a given allocentric direction, since entorhinal cortex cells responding at greater distances from a boundary have now been found (Koenig et al., 2011). Taken together these findings emphasize the importance of environmental boundaries to the hippocampal place cell representation of location within an environment. In this view, one can think of place cell firing as reflecting the match between the distances to boundaries around the animal and the distances and directions to which its BVC inputs are tuned (see Burgess et al., 1997; Hartley et al., 2000).

It is important to note that place cells are not solely driven by sensory information. For example, after a rat has experienced an environment, many of the cells continue to fire within their place field when the lights are tuned out (O'Keefe, 1976). Further compelling evidence for the mnemonic properties of place cells comes from experiments in which the environmental cues controlling place fields have been identified and it was then shown that place fields were maintained following the removal of these cues (Muller and Kubie, 1987; O'Keefe and Speakman, 1987; Save et al., 2005). For example, O'Keefe and Speakman (1987) recorded place cells whilst rats performed a "plus maze" task. Distant orienting cues predicted the rewarded locations and occasionally both

\section{A Predicting place cell firing in new environments}

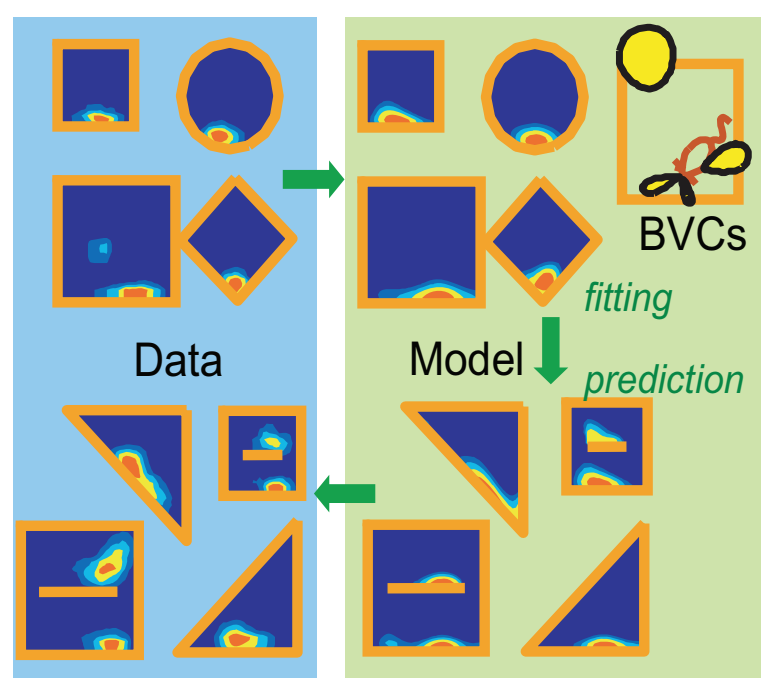

FIGURE 1 | The boundary vector cell (BVC) model of place cell firing Hartley et al. (2000); O'Keefe and Burgess (1996). (A) The likely BVC inputs to a place cell can be inferred from its firing pattern across environments of different shape (left). This can be used to predict its firing pattern in new

\section{B Examples of real boundary vector cells}

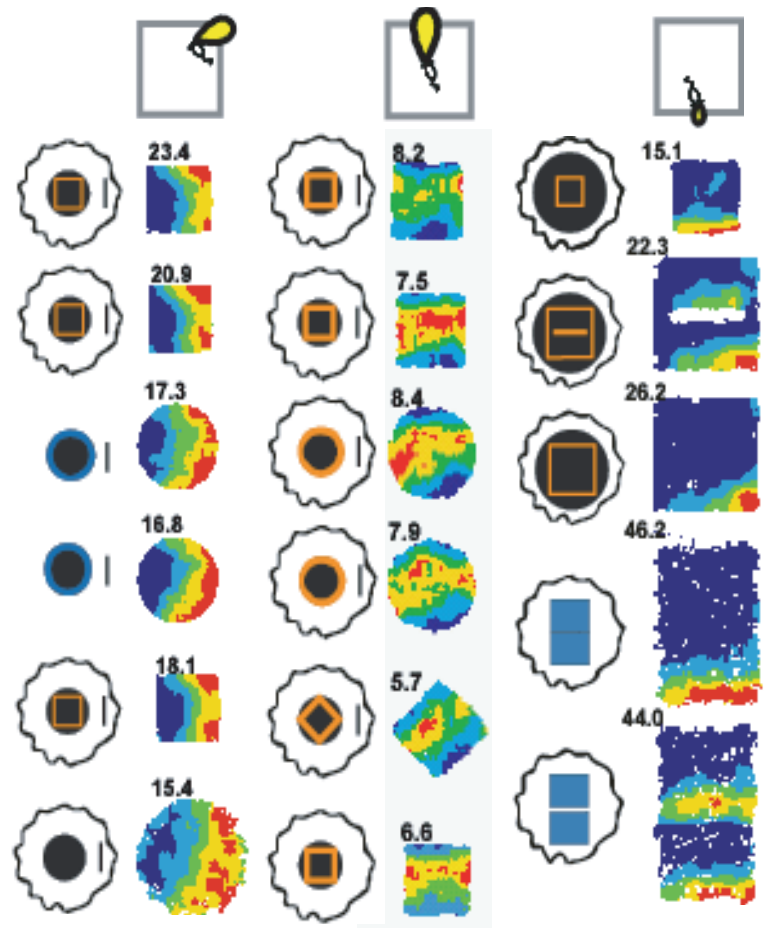

environmental shapes (right). (B) BVCs have now been discovered: the firing patterns are shown for three BVCs across a range of environments, including environmental changes which cause place cell remapping, adapted from Lever et al. (2009). 
the cues and the reward location were rotated, to ensure that the rats used the orientation cues to solve the task. Importantly, place fields rotated consistently with the cues. On some trials the cues were removed, either before or after the rat was placed in the maze. In the latter trials, the rat could perform the task based on its memory for the cue locations, whereas on the former trials it could only guess the correct location. Critically, the place cell ensemble maintained their place fields in a manner consistent with the animal's choice of target location on both trial types.

These studies show that place cells do not simply fire as a result of the sensory inputs impinging on them as a result of physically being present in an environment. Rather, the cells are encoding the more abstract concept of a place, in other words, a mental representation of where the rat "thinks" it is. We speculate below, that such representations are needed for mental imagery, which necessarily requires imagining being in a different place to one's current location (see Becker and Burgess, 2001; Burgess et al., 2001).

It is also important to note two further aspects of place cell firing. First, in addition to spatial location within an environment, place cells appear to signal which environment the animal is in. Thus, the place cell representations of two very different environments are radically different, or "remapped" (e.g., Bostock et al., 1991). Thus, if a rat is given enough experience of two perceptually similar environments, e.g., only differing in shape, the initially similar place cell representation of both environments (consistent with the BVC model) begins to distinguish the two environments (Lever et al., 2002). In addition, if moved into a completely novel environment (i.e., differing from the old one in smell, texture, and appearance), a completely different pattern of place cell firing is quickly established (Wills et al., 2005), see Fyhn et al. (2007) and Jeffery et al. (2004) for further discussion of the intricacies of place cell remapping. It may well be that, by remapping, the place cell ensemble signals when two environments are considered sufficiently different to representing a change in "context," i.e., a change in the expectancy of the events or stimuli likely to be encountered.

Second, place cell firing within a given environment is also modulated by non-spatial information. For example, some cells increase their firing in a specific location when the animal is engaged in a particular type of behavior (such as running or sniffing). Others fire maximally when an animal unexpectedly finds (or fails to find) an object in a particular location (O'Keefe, 1976). The modulation of firing rate in the place field appears to be independent of the spatial properties of firing (Huxter et al., 2003). The particular trajectory of movement through a place field can also influence its firing (Shapiro et al., 1997), especially in situations where multiple over-learned trajectories are present. The modulation of place cell firing by non-spatial factors demonstrates that these cells do not simply represent location, but also additional information about what happened in that location.

All of the above non-spatial, possibly experience-dependent, influences modulating the firing of place cells may contribute to the temporal variation in place cell firing occurring in parallel to the spatial variation (Manns and Eichenbaum, 2009; MacDonald et al., 2011). These factors may also contribute to the observation of extreme run-by-run variability in place cell firing (Fenton and Muller, 1998). With regard to these findings, it is interesting to note that variation of location in the vertical dimension also strongly modulates place cell firing (Hayman et al., 2011), especially when rodents rear and explore the walls of the environment, which is usually not tracked by standard recording equipment (Muller et al., 1987).

Finally, we note the existence of two other important spatial representations. Head-direction cells which are found along Papez's circuit from the mamillary bodies through the anterior thalamus, presubiculum, and entorhinal cortex, encode the animal's current head direction (Taube, 1998). Each cell fires when the head points in a specific allocentric direction, irrespective of the animal's location, so that the population of cells provides a compass-like signal. Grid cells, which are found in entorhinal cortex (Hafting et al., 2005) and pre- and para-subiculum (Boccara et al., 2010), fire whenever the animal enters any one of several locations in its environment. Interestingly, the firing locations of a grid cell lie at the vertices of an amazingly regular triangular grid across the environment, with different cells having grids which may have a different offset or scale relative to the environment, but tend to have the same orientation. It is thought that the population of grid cells supports path integration: updating their firing patterns in response to self-motion (e.g., McNaughton et al., 2006). The orientations of the place, grid, and head-direction cell representations appear to be linked in that they all rotate coherently when, for example, distal visual cues to orientation are rotated.

\section{A COMPUTATIONAL MODEL OF SPATIAL REPRESENTATION IN HUMANS}

The BVC model of place cell firing has been used as a starting point to define the functional architecture of human spatial cognition and imagery (Becker and Burgess, 2001; Burgess et al., 2001; Byrne et al., 2007). The basic idea is that a partial cue concerning the surrounding boundaries and visual textures of a familiar environment can cause reactivation of the complete place cell representation corresponding to a single location. The recurrent connections between place cells in area CA3 constrain activity there to remain within the subset of patterns of activity consistent with being in a single location. These patterns of activity form a continuous attractor within the space of possible patterns of activity, so that patterns of activity can move smoothly between the representations of different locations, while avoiding patterns of activity corresponding to being in more than one place simultaneously (the vast majority of possible patterns). See Zhang (1996) and Samsonovich and McNaughton (1997) for computational models, and Nakazawa et al. (2002) and Wills et al. (2005) for experimental support. The place cells then reactivate the complete representations of boundaries and visual textures via reciprocal connections back to parahippocampal and perirhinal cortices.

To allow imagery, the allocentric BVC representation (specifying layout in terms of North, South, etc.) must be translated into egocentric coordinates (i.e., left, right relative to the head). This translation is proposed to occur in posterior parietal and retrosplenial cortices, where neurons encode locations in combined egocentric and allocentric reference frames (Galletti et al., 1995; Snyder et al., 1998). The translation process requires the 
current (or imagined) viewing direction, which is specified by the head-direction cells found along Papez's circuit (Taube, 1998). The final egocentric image is supported by medial parietal areas (see Figure 3: green and black components).

Thus, at a more abstract level, the place cells can be thought of as constraining the activation of information in medial temporal neocortical areas to self-consistent subsets that correspond to being in a single location. For example, when imagining being at a concert, if the orchestra is ahead of you, then the circle must be behind you, the exit to your left, etc. See Recce and Harris (1996) for related discussion of the relationship between place cell firing and Marr's (1971) model of associative memory. The requirement for specification of a viewing direction (by head-direction cells), provides one potential role for (extra-hippocampal) Papez's circuit in recollection, and its impairment in patients with damage to it (e.g., Tsivilis et al., 2008).

The imposition of a viewpoint location (via place cells) and viewing direction (via head-direction cells) on the products of retrieval from long-term (allocentric) memory stores allows the creation of spatially coherent (egocentric) scenes in medial parietal areas. Beyond this, imagery should allow dynamic movement of viewpoint, as when imagining the sequence of events in an episode or journey. The model proposed a mechanism for updating the viewpoint position by motor efference signals in the case of actual movement, or by mock motor efference signals generated by medial prefrontal areas in the case of imagery and planning. The original mechanism (Becker and Burgess, 2001; Burgess et al., 2001) proposed that the egocentric-allocentric translation mechanism could also be used to perform the process of spatial updating. However, the subsequent discovery of grid cells (Hafting et al., 2005), and their association with path integration, suggests that grid cells might support this process instead.

How does our model compare to other models? There are many characterizations of the roles of different regions of the medial temporal lobes in memory (e.g., Aggleton and Brown, 1999; Eacott and Gaffan, 2005; Eichenbaum et al., 2007; Mayes et al., 2007). The prevailing view is that the perirhinal cortex is particularly specialized for representing objects, the parahippocampal cortex is specialized for representing spatial "contexts" or places, and the hippocampus binds together outputs from both these areas to represent episodes as a whole (the objects in the correct spatial contexts). Computational models similarly locate the hippocampus as the region responsible for binding the output of different cortical regions into a unified representation of all of the facets that constitute an episode (e.g., Marr, 1971; Teyler and DiScenna, 1986; McClelland et al., 1995; Rolls and Treves, 1997; Norman and O'Reilly, 2003). One specific proposal of this type, that looks beyond purely mnemonic processing, is that the hippocampus is specialized for the formation of associations between disparate elements of an episode, occurring across space and time and in the flexible manipulation of learned relationships among items (the relational memory theory; Cohen and Eichenbaum, 1993; see also Olsen et al., 2012 and Zeithamova et al., 2012).

Our conceptualization of hippocampal function shares obvious similarities with these theories, particularly regarding the representations supported by the perirhinal and parahippocampal cortices. Our goal is not to refute these models, but to specify more precisely the neural representations supported by the hippocampus, and to explain how and why the hippocampus binds or relates together the output of some of these neocortical regions. First, when imagining being in an environment, the hippocampus is necessary to locate oneself within the imagined scene and to represent the shape of the environment with respect to extended boundaries. Thus, the hippocampus is required to form mental images of scenes that are spatially coherent rather than simply stereotyped lists of the content of a scene. Second, when visualizing a scene, reciprocal connections between place cells and cells representing objects in perirhinal cortex constrain the retrieval of objects that are consistent with being in a specific location within the environment (Burgess et al., 2001; Byrne et al., 2007). Thus, place cells enable efficient search through the mass of abstract viewpoint-independent information stored elsewhere in the cortex. At the end of the next section we will discuss the similarities and distinctions between our model and relational processing theories of hippocampal function.

\section{EVIDENCE FOR SPATIAL REPRESENTATIONS IN HUMANS}

A wealth of studies have demonstrated that the hippocampus is necessary for spatial memory in humans (Smith and Milner, 1981; Abrahams et al., 1997; Bohbot et al., 2002; King et al., 2004), see Burgess et al. (2002) for a review. Nevertheless, more specific studies are necessary to demonstrate that humans exhibit behavior consistent with the use of place-cell-like representations of space. The particular importance of the hippocampus in representing topographical layout was demonstrated by Hartley et al. (2007). In this study, patients with hippocampal damage were asked to recognize mountain landscapes from different viewpoints across short delays. In the spatial subtests, the topography of the landscape remained constant but the lighting and color of the vegetation was altered (simulating changes in the time of day and the seasons). In the non-spatial subtests participants were required to ignore the topography but match the lighting and vegetation. The patients were all impaired on the topographical subtests but not the non-spatial control tasks which were closely matched for performance in young controls. Lee and colleagues have reported similar findings (Lee et al., 2005a,b).

More direct evidence that humans use place-cell-like representations of space in spatial memory comes from a virtual reality study by Hartley et al. (2004). Participants were required to remember the location of a flag encountered within a rectangular arena surrounded by distal cues for orientation. In the test period they moved to where they thought the flag had been located. On some trials, the dimensions of the environment were altered during the delay between study and test, in a similar manner to the study of O'Keefe and Burgess detailed above. The spatial distribution of responses were better predicted by a model assuming that the location was encoded by BVCs than by alternative models assuming representation of the location in terms of the angles to the corners, or the distances to walls etc.

Place cell firing is influenced by environmental boundaries more so than intramaze landmarks (cf. O'Keefe and Burgess, 1996; Cressant et al., 1997). Similarly, the human hippocampus is involved in learning locations relative to a boundary wall than 
relative to a landmark within the arena bounded by that wall. Doeller et al. (2008) asked participants to remember the locations of objects presented within a circular arena in the presence of a landmark cue (a traffic cone). To ensure that participants attended to both task-relevant cues (the boundary and the landmark), on some trials these cues moved relative to each other and half of the objects moved to a target location consistent with the boundary wall whilst the other half moved to a target location consistent with the intramaze landmark. Learning relative to the boundary wall correlated with hippocampal activation whereas learning relative to the landmark correlated with the activation in the dorsal striatum and parietal lobe.

These studies all suggest that humans do indeed use similar, hippocampally mediated, spatial representations to rodents. However, some studies have provided more direct evidence for this. In a virtual reality navigation task, Ekstrom et al. (2003) described single units in patients with epilepsy that had properties similar to place cells, and were clustered in the hippocampus. In a related finding, Morgan et al. (2011) used fMRI scanning while participants looked at pictures of a familiar environment. They found fMRI "adaptation" in the hippocampus according to the physical proximity of the locations from which the pictures were taken, consistent with a neuronal representation of environmental location in the hippocampus.

Unlike place cells (Redish et al., 2000; Dombeck et al., 2010), the firing patterns of grid cells show some topographical organization. Simultaneously recorded grid cells have grid-like firing fields with the similar orientations in environmental space (Hafting et al., 2005; Barry et al., 2007). Thus, the neuronal firing dynamics within entorhinal cortex might differ according to whether the participant (in a virtual environment) is running either aligned or misaligned to the three principal directions of the grids. The predicted 6-peaked modulation of fMRI signal as a function of running direction was observed in human entorhinal cortex (Doeller et al., 2010). Intriguingly, a similar pattern was seen throughout the autobiographical memory system. We might speculate that grid cells allow dynamic translation of the viewpoint used to construct imagery, consistent with their role in path integration.

The studies described above all support the view that humans use similar neural representations of space as rodents. However, we are still left with the question, are these representations actually used for mental imagery? Direct evidence that the hippocampus is necessary for imagining environments comes from a study by Hassabis et al. (2007b). A group of patients with hippocampal damage were asked to imagine themselves in a large-scale space (such as on a beach) and describe what was around them. As a group, the patients' descriptions were poorer in detail than the controls', and particularly lacked spatial coherence. When healthy adults performed this task in an fMRI experiment, the hippocampus was activated (Hassabis et al., 2007a), see also Addis et al. (2007).

Whilst the studies of Hassabis and colleagues point to a contribution of the hippocampus to imagination, they do not test a specific model of what this contribution might be. We set out to test a specific prediction of the model discussed above in an imagery study using fMRI (Bird et al., 2010). Under the BVC model of place cell firing, the influence of a given object (a landmark or a boundary) on the place cell representation of location is proportional to the horizontal angle subtended by it at the participant. Accordingly, increasing the number of enclosing environmental boundaries should boost the contribution of the hippocampus to orientation within that environment. The model makes the same prediction for imagined environments. If we can engineer a situation whereby we can manipulate the number of enclosing boundaries in the environments that participants imagine standing within, then we should be able to observe the effect of imagining more or fewer boundaries on the hippocampal BOLD response.

The experiment is summarized in Figure 2. Participants were shown aerial views of simple environments made of walls and towers and then required to imagine standing within the environments. We varied parametrically the number of enclosing walls in the imagined scenes, while keeping the overall number of structural elements constant. The experiment also included a non-spatial, difficulty control, were we varied the colors of the structural elements. Both the numbers of boundaries and the color complexity affected task difficulty. Interestingly, the conditions with intermediate numbers of boundaries were reliably the most difficult to imagine, indicating that participants found correctly imagining the configurations of towers and walls more complex than scenes containing mostly towers or mostly walls. Despite this, there was a significant parametric effect of increasing numbers of boundaries in the hippocampus but no correlation with increasing spatial complexity of the scenes.

We will briefly consider these findings with respect to other conceptualizations of hippocampal function that have been advanced in this Special Issue. Similarly to our model, several current theories stress the particular types of representation and processing supported by the hippocampus. In this respect, both these theories and our model differ from theories that stress the type of memories that that the hippocampus support, for example, that the hippocampus has a specialized role in long-term declarative memory or in consciously accessible memories (Cohen and Squire, 1980; Moscovitch, 1995; Squire et al., 2004; see Hannula and Greene, 2012). One of the most popular positions is that the hippocampus is specialized for relational processing. This theory can be applied to a wide range of materials whether conscious or non-conscious, and to tasks requiring online guidance of behavior as well those involving a delay. For example, hippocampally mediated representations of the relationships between items may be important for aspects of linguistic processing (Duff and Brown-Schmidt, 2012; see also O'Keefe and Nadel, 1978) and reasoning (Zeithamova et al., 2012). Nevertheless, it remains a challenge to specify precisely which representations and which relations require hippocampal support, and how they might correspond to the "elements" of an episodic memory. Some authors have attempted to clarify this (Cohen et al., 1999; Mayes et al., 2007), but it is not clear whether these definitions are universally accepted by adherents of the theory.

Both ourselves and Lee et al. (2012) have focussed on the role of the hippocampus in spatial processing, for which some of these questions can be addressed with more specificity. For example, O'Keefe and Nadel (1978) suggest that the hippocampus is 


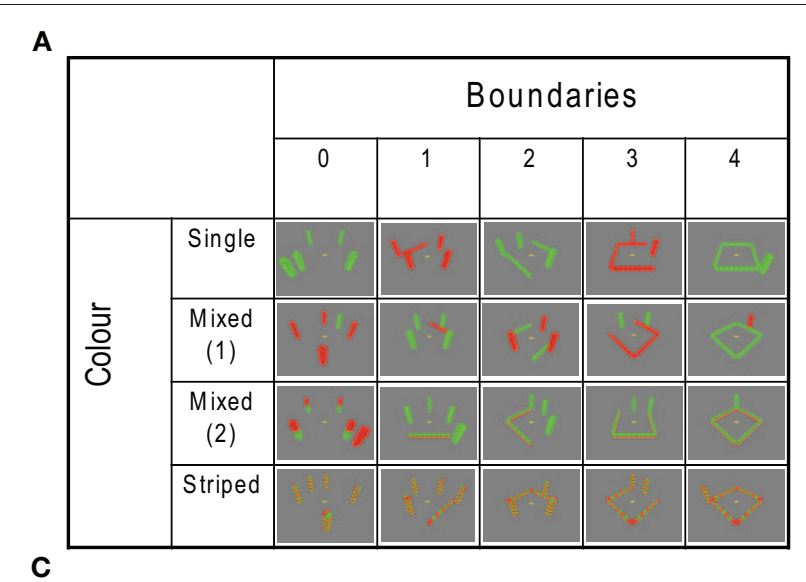

C

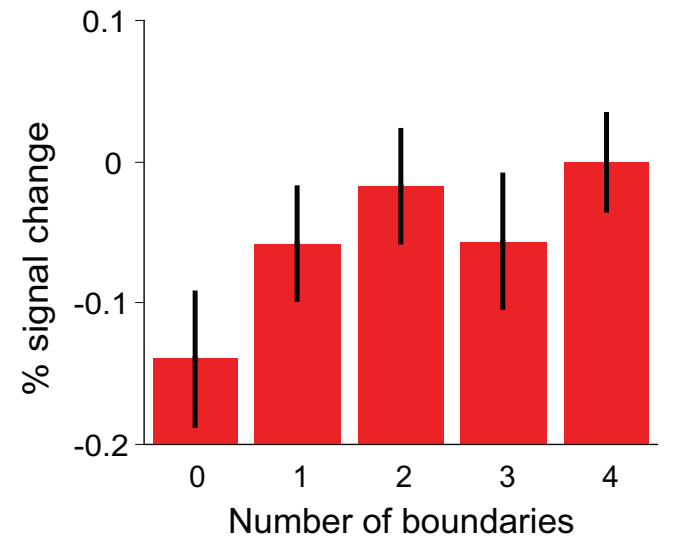

FIGURE 2 | In this fMRI study, participants were asked to imagine standing in various different environments containing combinations of boundaries (walls) and towers (non-boundary environmental elements) and turning through $360^{\circ}$. Aerial views of the environments used are shown in panel (A). The number of enclosing boundaries was varied between 0 and 4, whilst the total number of boundaries and towers was always 5 . Complexity was also manipulated by varying the colors of the boundaries and towers. Panel (B)
B

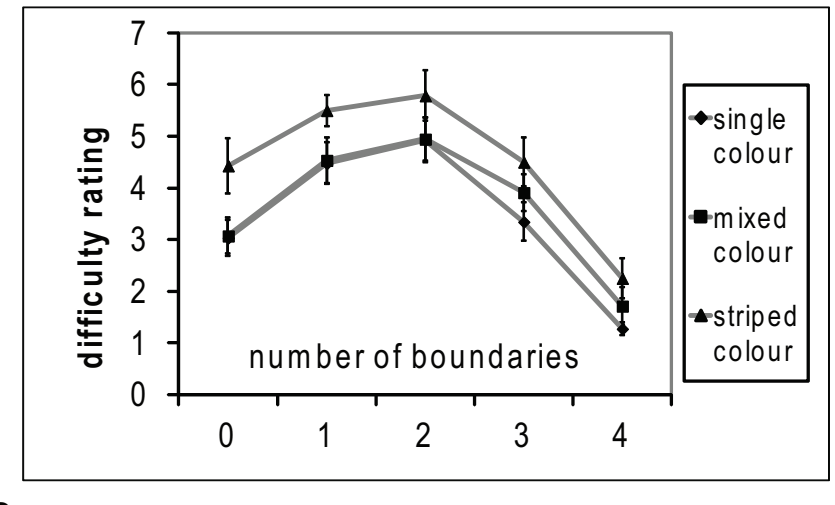

D

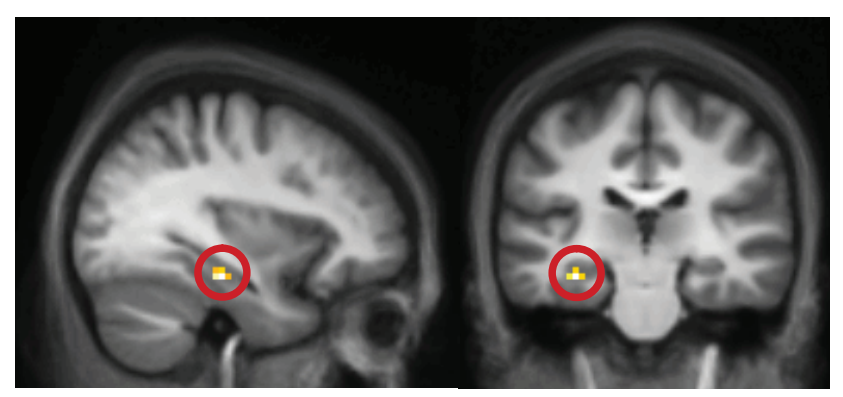

specifically concerned with flexible representations of space, i.e., those that allow a particular location to be found from different starting points and independently of rigid (habitual) routes or learned responses to individual stimuli. The same idea of flexibility is incorporated into the idea of relational memory, and generalized beyond the spatial domain (Cohen and Eichenbaum, 1993). The independence from individual stimuli is taken up by Lee et al., who suggest that "the hippocampus processes complex conjunctions of spatial features," see also Sutherland and Rudy (1989).

In this review and elsewhere, we have tried to further specify which conjunctions of spatial features are supported by the hippocampal formation, and how they are used to support memory, imagery, and navigation. By attempting to understand spatial processing from a neuronal level, we can show that some "elements" of a scene are preferentially processed by the hippocampus (e.g., environmental boundaries versus local landmarks; Doeller et al., 2008; Bird et al., 2010), and that some type of processing are favored (e.g., incidental versus reinforcement learning, Doeller and Burgess, 2008, see also Tolman, shows ratings of how difficult each condition was to imagine; intermediate numbers of boundaries and the striped colored conditions were rated most difficult. Panel (C) shows a parametric modulation of activity in a region of the left hippocampus with increasing numbers of boundaries in the imagined scenes. This region is shown in (D). These results are consistent with the hippocampus representing location within an imagined environment with respect to extended environmental boundaries.
1948; Hirsch, 1974; O'Keefe and Nadel, 1978). In the imaging study detailed above (Figure 2), a purely relational processing account might have predicted greatest hippocampal activity when imagining scenes comprising roughly equal numbers of walls and towers, since these were most difficult to imagine. However, no regions within the hippocampus showed this pattern, whereas, a region of hippocampus did show a correlation with increasing numbers of environmental boundaries, consistent with our model. A challenge for the future is to make similarly detailed predictions regarding other types of hippocampally mediated relational processing such as relating items across time.

In summary, the studies we have presented provide a strong case for the role of the hippocampus in both the retrieval of information to construct mental imagery, and in constraining the resultant image to be spatially coherent. The combination of rodent and human data support a model whereby retrieval reflects place cell firing in the hippocampus, which reinstates the (spatial) contextual characteristics of an event via activation of parahippocampal and perirhinal cortices. The 
key aspect of retrieval via place cell activity is that the potentially myriad products of retrieval are constrained to all be consistent with observation from the same viewpoint location, ensuring that images are constructed in a spatially coherent manner. These (allocentric) medial temporal representations can then form an egocentric projection in medial parietal areas, given imposition of a viewing direction provided by head-direction cells. This latter step requires processing by "gain field" neurons in parietal cortex and parieto-occipital sulcus. The end product of this reconstructive process allows boundaries and objects in spatial configurations consistent with a single specific viewpoint to be experienced as egocentric imagery.

\section{LOST HIPPOCAMPAL CONTROL AND WHEN “IMAGERY GOES WRONG"}

In addition to providing a neural-level explanation of the processes of imagery and retrieval in healthy memory, we can use the model to generate predictions about the way in which imagery can go wrong if the hippocampal provision of contextual support is lost or weakened. One example of when imagery can "go wrong" involves memory for traumatic events. Following the experience of trauma, memories for the episode can intrude into an individual's consciousness in the form of distressing sensorybound images, as in PTSD. In this section, we extend the model and detail the way in which different representational systems for an event can be affected in different ways and, specifically, how down-regulation of the hippocampus within this model might contribute to involuntary retrieval of imagery. This view is consistent with the "dual representation" account of intrusive imagery development following a traumatic event (see Jacobs and Nadel, 1985; Brewin et al., 2010).

In the spirit of the dual representation account, the information in an event can be encoded in two different ways. As detailed above, long-term allocentric contextual representations (or "C-reps") are encoded in the hippocampus and surrounding medial temporal lobe (Figure 3: green components). In addition, short-term egocentric representations of the sensory and affective aspects of an event are supported by a second system, including the amygdala, insula, and sensory association areas. These images, comprising central sensory/perceptual features of an experience provide a sensory-bound representation, or S-rep, of a scene that is dependent on the perceiver's viewpoint (Figure 3: red components).

For a neutral event, initial sensory-based representations will become relatively inaccessible as they quickly decay. Over time, C-reps will become integrated within semantic and autobiographical memory via hippocampal and neocortical interactions (Marr, 1970, 1971; McClelland et al., 1995), providing a basis for the generation of meaningful interpretations and the construction of novel images through recombination of past objects and scenes. Egocentric imagery can be generated in medial parietal areas from C-reps, via the process of allocentric to egocentric translation, as described above. If lower-level sensory information is retained, it could be retrieved via associations with the egocentric image, providing more vivid sensory and affective quality to imagery. Retrieval, whether voluntary or involuntary, and the construction of mental imagery are under top-down control, modulated by the hippocampus and its connections from prefrontal areas.

\section{TRAUMA-RELATED ALTERATIONS IN MEMORY REPRESENTATIONS AND RELATED IMAGERY}

Although initial egocentric imagery is expected to rapidly decay as more stable allocentric representations are encoded in the hippocampus and medial temporal lobe, in some situations these less flexible images might persist for longer. Alterations in stress responses during an event provide one instance where such representations might actually be enhanced. It is well established that the experience of an emotional event can facilitate memory (Cahill and McGaugh, 1998). During a stressful episode, immediate interoceptive representations of bodily responses become associated with sensory information of a scene, mediated by insular cortex and amygdale (LeDoux, 1996; Craig, 2002, 2009; Critchley et al., 2004), creating a stronger and more enduring S-rep. The storage of a corresponding C-rep with the S-rep enables its accessibility to voluntary retrieval and allows the integration of event-related information into semantic and autobiographical memory systems. Intact C-reps provide context dependency of event-related information and the prevention of spontaneous reactions to environmental stimuli through inhibitory prefrontal pathways, modulated by the hippocampus (Kim and Fanselow, 1992; Anagnostaras et al., 1999; Anderson et al., 2004). The reconstruction of mental imagery from a transient stressful event would be facilitated, resulting in the activation of strengthened S-reps top-down via corresponding higher level representations, experienced as egocentric imagery from the same viewpoint in the precuneus.

For imagery to "go wrong" and become liable to spontaneously intrude into consciousness, an extreme level of stress would be required to disrupt hippocampal function (e.g., Kim and Diamond, 2002; Payne et al., 2007). Down-regulation of the hippocampus will result in an impoverished C-rep. In contrast, physiological reactions of the event that contribute to S-reps are strengthened via simultaneous increases in amygdala activity (LaBar et al., 1998). The resulting imbalance of an enduring S-rep and impoverished C-rep creates a persistent emotion-laden mental image of the scene without a coherent spatial context. Voluntary retrieval and the ability to construct mental imagery in a controlled way are impaired due to the decrease in hippocampal support and its mediation of top-down control. S-reps can therefore, be triggered involuntarily through the experience of sensory inputs in the environment that share similarities to characteriztics of the original event. Reactivation of an S-rep without its corresponding C-rep results in the re-experience of vivid egocentric imagery and associated affective and physiological reactions. These representations would suffer an inability to integrate information into long-term memory due to the absence of robust associated C-reps. It should be noted that damage to the hippocampal system alone would not result in intrusive imagery. For this to occur, an impoverished C-rep must be accompanied by the creation of an enduring S-rep through an extreme event such as a trauma. Interestingly, some cognitive behavioral therapies for remediation of PTSD can be seen as attempts to (re)establish a 


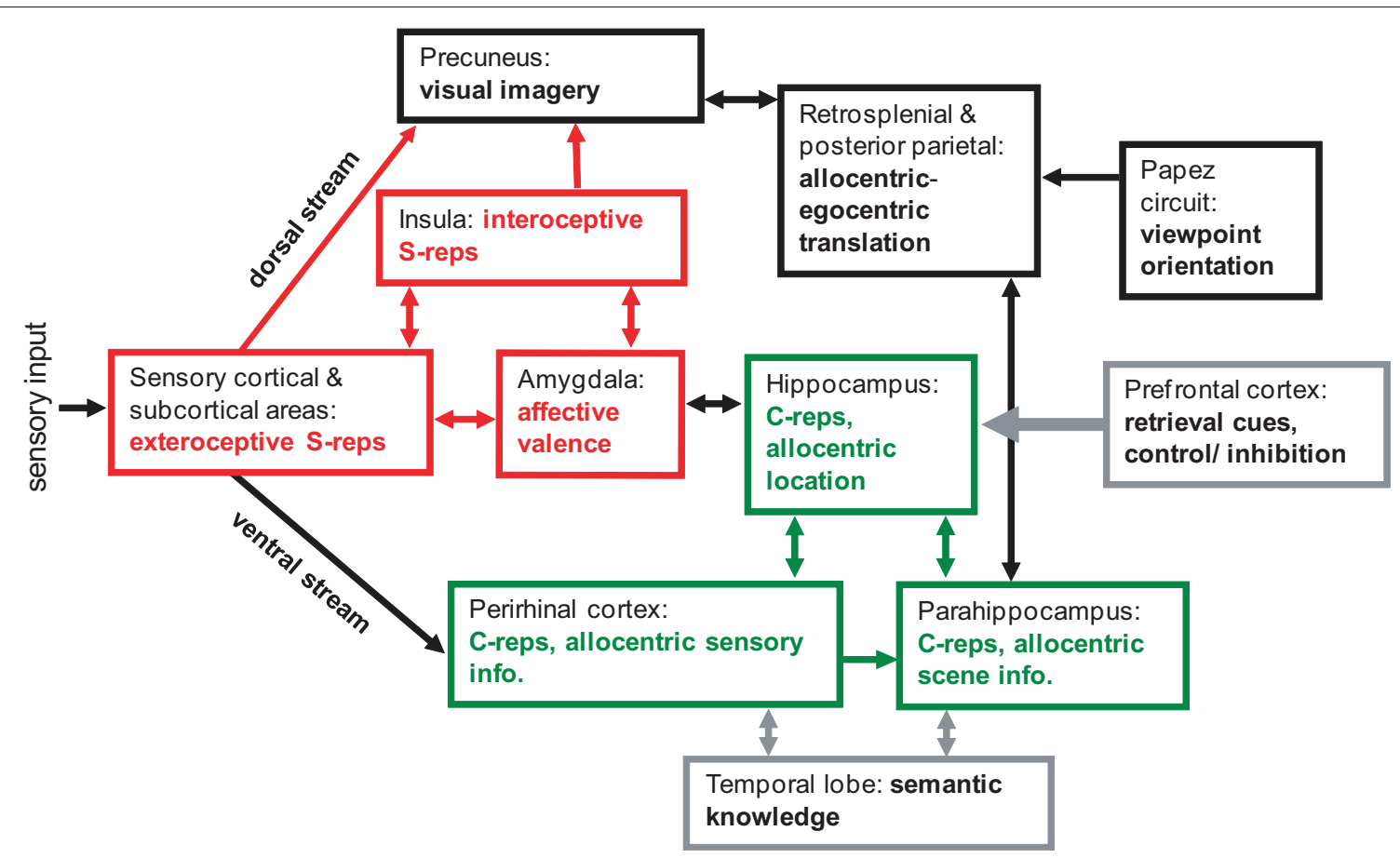

FIGURE 3 | A schematic model of memory and imagery, showing the approximate regions and pathways involved in, and supporting, abstracted contextual representations (C-reps, in green) and sensory bound representations (S-reps, in red). Scenes with strong affective content create enduring S-reps supported by amygdale, sensory areas and insula. S-reps are normally reactivated voluntary via a corresponding C-rep, but strong S-reps with weak association to C-reps can cause involuntary intrusive imagery. robust C-rep in association with a traumatic S-rep, see Brewin et al. (2010) for further discussion.

\section{EVIDENCE RELATING INTRUSIVE IMAGERY TO AN IMBALANCE IN MEMORY REPRESENTATIONS}

To directly assess the way in which the balance between egocentric and allocentric representations of an event might contribute to intrusive imagery, we devised a study to investigate spontaneous imagery experiences following exposure to stressful material and concurrently assessed egocentric and allocentric memory performance in the same individuals (Bisby et al., 2010). We utilized an analog trauma paradigm as a method to assess intrusive imagery, which involves the presentation of film clips from real-life traumatic events. Participants are required to record any intrusive imagery of the footage over the following week and then return for a test of explicit recall (see Holmes and Bourne, 2008). We used alcohol as a pharmacological tool to dissociate memory processes. Acute administration of alcohol has been shown to disrupt hippocampal-dependent memory in rodents (Matthews et al., 1995; White et al., 1998) and humans (Curran and Hildebrandt, 1999; Leitz et al., 2009). In a previous study assessing intrusive imagery following encoding of a stressful film, alcohol administered prior to watching the film was shown to affect intrusive imagery in different ways at different doses (Bisby et al., 2009). Individuals administered a low dose of alcohol were found to record significantly more intrusive images compared to those given a placebo drink, whereas participants in the high dose alcohol group showed a reduction in the number of intrusive images. It is interesting to note that explicit memory testing after one week followed a different pattern of results, with decreasing memory performance as alcohol dose increased.

The balance between allocentric and egocentric memory was measured by examining viewpoint dependence within a virtual reality spatial memory task (King et al., 2002, 2004). In this task, participants encode object locations within a virtual environment and memory is tested from the same-viewpoint as encoding or from a shifted-viewpoint. Shifted-view performance requires an intact allocentric representation, presumably supported by the hippocampus and surrounding structures. In contrast, same-view recognition can be solved by an egocentric representation of the scene through recognition of object locations relative to the perceiver's original viewpoint. Support for a role of the hippocampus in this task has been provided by findings from a patient with focal bilateral hippocampal pathology (Jon: Vargha-Khadem et al., 1997), who was unable to recognize object locations from a shifted-viewpoint, whereas same-view performance was intact (King et al., 2002, 2004).

The results from this study are summarized in Figure 4. We replicated previous findings showing an alcohol-induced dose-dependent inverted U-shaped curve on intrusive imagery. Participants administered low dose alcohol prior to encoding the traumatic material showed a significant increase in the number of intrusive images experienced following exposure to the stressful material, whereas the high dose group showed no such increase. Further, explicit recall of the footage was affected in a linear 


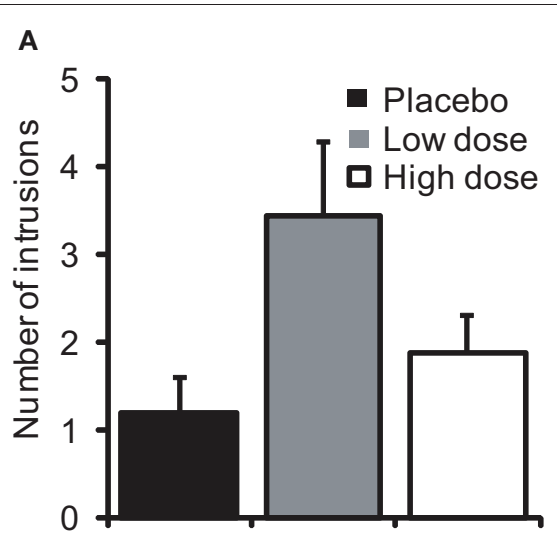

C

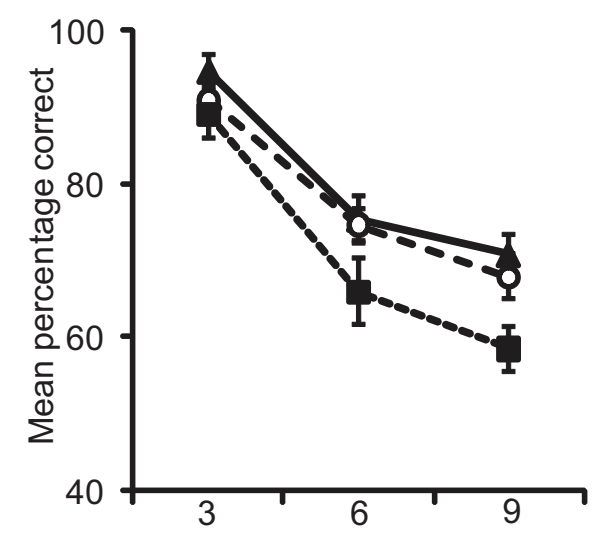

B
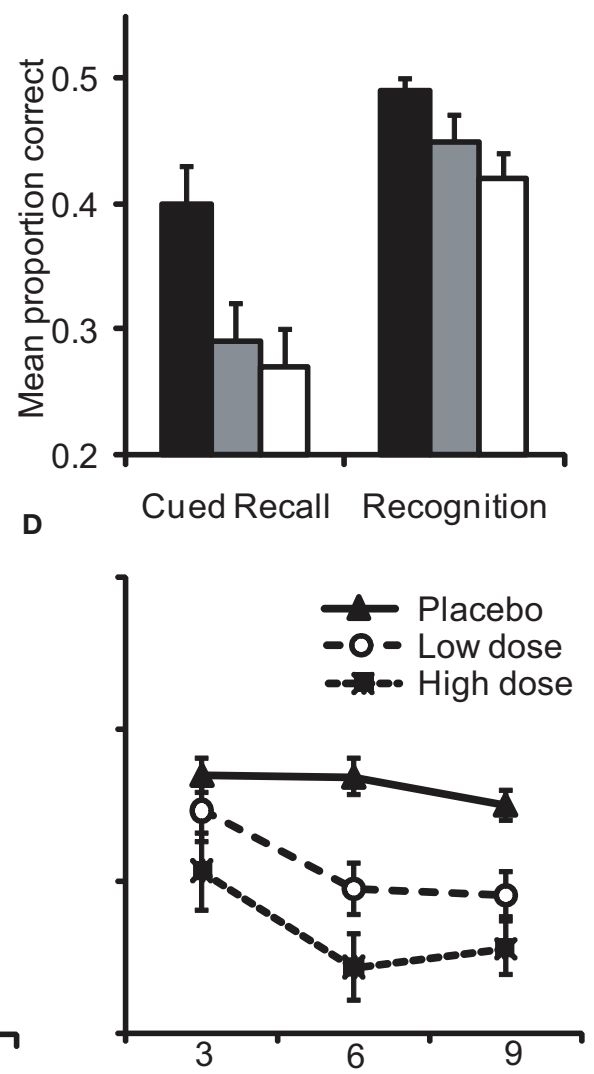

Listlength

FIGURE 4 | Alterations in (A) the number of intrusive images reported by participants over 7 days following exposure to a stressful film and $(B)$ explicit memory for the footage on day 8 as a function of alcohol dose. Object location recognition tested from the (C) same-view as encoding was spared under low dose alcohol but impaired by high dose, whereas (D) shifted-view recognition was impaired following the administration of low and high doses ladapted from Bisby et al. (2010)]. manner following alcohol with greater decreases in the number of items remembered as dose increased. Results on viewpointdependent memory performance were complementary, with the low dose group showing a selective reduction in shifted-view object location recognition and spared same-view performance. In those participants administered high dose alcohol, performance was impaired on both same- and shifted-view conditions of the task, demonstrating a global impairment in memory. Interestingly, in individuals with intact egocentric memory, evidenced by high same-view recognition performance, decrements in shifted-view recognition were correlated with increases in intrusive imagery experiences. Overall, these findings support a model whereby intrusive imagery occurs due an imbalance in C-reps and S-reps during exposure to a stressful event.

\section{SUMMARY}

To attempt to form a mechanistic neural-level model of the function of the hippocampus, we took inspiration from the detailed spatial correlates of neuronal firing in and around the hippocampus, Papez's circuit and posterior parietal cortices. The properties of these cells, how they represent spatial location, environmental boundaries, spatial orientation, and viewing direction, indicate a very specific model for how spatial information is retrieved and processed to form mental imagery for scenes (see Becker and Burgess, 2001; Burgess et al., 2001; Byrne et al., 2007). In the first half of the paper we reviewed this model and some of the supporting experimental evidence gathered since its original proposal. The model is not necessarily incompatible with other psychological models of the hippocampal role in memory or imagery but, by considering the constraints and information provided by spatial paradigms, it provides a very specific description of the neural mechanisms and representations involved.

In the second half of the paper, we outlined a recent extension of the model (Brewin et al., 2010) which aims to provide a neurophysiological implementation of the dual representation model of PTSD (see Jacobs and Nadel, 1985; Brewin et al., 2010). This model makes several predictions at the neuronal and behavioral levels. We review evidence for one of these predictions (Bisby et al., 2010); that intrusive imagery follows from encoding conditions in which (presumably hippocampal-dependent) allocentric spatial representations are impaired relative to egocentric spatial 
representations. A neuronal prediction would be that decreased hippocampal activation at encoding leads to increased subsequent intrusive imagery, in contrast to the well-known effect of increased hippocampal activation at encoding leading to increased declarative memory (e.g., Davachi and Wagner, 2002).

In summary, single unit recordings from rodents in spatial paradigms provides enough detailed information concerning the relationship of neuronal firing to behavioral variables to be able to describe a specific neuronal model of processing in and around the hippocampus. We have used such a model to investigate the contribution of the hippocampus to retrieval and imagery for spatial scenes. The results imply that the hippocampus ensures a unique and common viewpoint for retrieved information: providing a coherent allocentric spatial context within which imagery can take place. In the absence of such a context, the encoding of strong (a-contextual egocentric) sensory and affective representations of traumatic events can lead to an increased incidence of subsequent intrusive imagery.

\section{ACKNOWLEDGMENTS}

We gratefully acknowledge grant funds from the Medical Research Council UK and the Wellcome Trust.

\section{REFERENCES}

Abrahams, S., Pickering, A., Polkey, C. E., and Morris, R. G. (1997). Spatial memory deficits in patients with unilateral damage to the right hippocampal formation. Neuropsychologia 35, 11-24.

Addis, D. R., Wong, A. T., and Schacter, D. L. (2007). Remembering the past and imagining the future: common and distinct neural substrates during event construction and elaboration. Neuropsychologia 45, 1363-1377.

Aggleton, J. P., and Brown, M. W. (1999). Episodic memory, amnesia, and the hippocampal-anterior thalamic axis. Behav. Brain Sci. 22, 425-490.

Anagnostaras, S. G., Maren, S., and Fanselow, M. S. (1999). Temporally graded retrograde amnesia of contextual fear after hippocampal damage in rats: within-subjects examination. J. Neurosci. 19, 1106-1114.

Anderson, M. C., Ochsner, K. N., Kuhl, B., Cooper, J., Robertson, E., Gabrieli, S. W., Glover, G. H., and Gabrieli, J. D. (2004). Neural systems underlying the suppression of unwanted memories. Science 303, 232-235.

Barry, C., Hayman, R., Burgess, N., and Jeffery, K. J. (2007). Experiencedependent rescaling of entorhinal grids. Nat. Neurosci. 10, 682-684.

Becker, S., and Burgess, N. (2001). A model of spatial recall, mental imagery and neglect. Neural Inf. Process. Syst. 13, 96-102.

Bird, C. M., Capponi, C., King, J. A., Doeller, C. F., and Burgess, N. (2010). Establishing the boundaries: the hippocampal contribution to imagining scenes. J. Neurosci. 30, 11688-11695.

Bisby, J. A., Brewin, C. R., Leitz, J. R., and Valerie, C. H. (2009). Acute effects of alcohol on the development of intrusive memories. Psychopharmacology (Berl.) 204, 655-666.
Bisby, J. A., King, J. A., Brewin, C. R., Burgess, N., and Curran, H. V. (2010). Acute effects of alcohol on intrusive memory development and viewpoint dependence in spatial memory support a dual representation model. Biol. Psychiatry 68, 280-286.

Boccara, C. N., Sargolini, F., Thoresen, V. H., Solstad, T., Witter, M. P., Moser, E. I., and Moser, M. B. (2010). Grid cells in presubiculum and parasubiculum. Nat. Neurosci. 13, 987-994.

Bohbot, V. D., Jech, R., Ruzicka, E. Nadel, L., Kalina, M., Stepankova, K., and Bures, J. (2002). Rat spatial memory tasks adapted for humans: characterization in subjects with intact brain and subjects with selective medial temporal lobe thermal lesions. Physiol. Res. 1 (Suppl. 51), S49-S65.

Bostock, E., Muller, R. U., and Kubie, J. L. (1991). Experience-dependent modifications of hippocampal place cell firing. Hippocampus 1, 193-205.

Brewin, C. R., Gregory, J. D., Lipton, M., and Burgess, N. (2010). Intrusive images in psychological disorders: characteristics, neural mechanisms, and treatment implications. Psychol. Rev. 117, 210-232.

Burgess, N., Becker, S., King, J. A., and O'Keefe, J. (2001). Memory for events and their spatial context: models and experiments. Philos. Trans. R. Soc. Lond. B Biol. Sci. 356, 1493-1503.

Burgess, N., Donnett, J. G., Jeffery, K. J., and O'Keefe, J. (1997). Robotic and neuronal simulation of the hippocampus and rat navigation. Philos. Trans. R. Soc. Lond. B Biol. Sci. 352, 1535-1543.

Burgess, N., Maguire, E. A., and O'Keefe, J. (2002). The human hippocampus and spatial and episodic memory. Neuron 35, 625-641.

Byrne, P., Becker, S., and Burgess, N. (2007). Remembering the past and imagining the future: a neural model of spatial memory and imagery. Psychol. Rev. 114, 340-375.

Cahill, L., and McGaugh, J. L. (1998). Mechanisms of emotional arousal and lasting declarative memory. Trends Neurosci. 21, 294-299.

Cohen, N. J., Ryan, J., Hunt, C. Romine, L., Wszalek, T., and Nash, C. (1999). Hippocampal system and declarative (relational) memory: summarizing the data from functional neuroimaging studies. Hippocampus 9, 83-98.

Cohen, N. J., and Squire, L. R. (1980). Preserved learning and retention of pattern-analyzing skill in amnesia: dissociation of know how and knowing that. Science 210, 207-210.

Cohen, N. J., and Eichenbaum, H. (1993). Memory, Amnesisa and the Hippocampal System. Cambridge, MA: MIT Press.

Craig, A. D. (2002). How do you feel? Interoception: the sense of the physiological condition of the body. Nat. Rev. Neurosci. 3, 655-666.

Craig, A. D. (2009). How do you feel-now? The anterior insula and human awareness. Nat. Rev. Neurosci. 10, 59-70.

Cressant, A., Muller, R. U., and Poucet, B. (1997). Failure of centrally placed objects to control the firing fields of hippocampal place cells. J. Neurosci. 17, 2531-2542.

Critchley, H. D., Wiens, S., Rotshtein, P., Ohman, A., and Dolan, R. J. (2004). Neural systems supporting interoceptive awareness. Nat Neurosci. 7, 189-195.

Curran, H. V., and Hildebrandt, M. (1999). Dissociative effects of alcohol on recollective experience. Conscious. Cogn. 8, 497-509.

Davachi, L., and Wagner, A. D. (2002). Hippocampal contributions to episodic encoding: insights from relational and item-based learning. J. Neurophysiol. 88, 982-990.

Doeller, C. F., and Burgess, N. (2008). Distinct error correcting and incidental learning of location relative to landmarks and boundaries. Proc. Natl. Acad. Sci. U.S.A. 105, 5909-5914.

Doeller, C. F., Barry, C., and Burgess, N. (2010). Evidence for grid cells in a human memory network. Nature 463, 657-661.

Doeller, C. F., King, J. A., and Burgess, N. (2008). Parallel striatal and hippocampal systems for landmarks and boundaries in spatial memory. Proc. Natl. Acad. Sci. U.S.A. 105, 5915-5920.

Dombeck, D. A., Harvey, C. D., Tian, L., Looger, L. L., and Tank, D. W. (2010). Functional imaging of hippocampal place cells at cellular resolution during virtual navigation. Nat. Neurosci. 13, 1433-1440.

Duff, M. C., and Brown-Schmidt, S. (2012). The hippocampus and the flexible use and processing of language. Front. Hum. Neurosci. 6:69. doi: 10.3389/fnhum.2012.00069

Eacott, M. J., and Gaffan, E. A. (2005). The roles of perirhinal cortex, postrhinal cortex, and the fornix in memory for objects, contexts, and events in the rat. Q. J. Exp. Psychol. B 58, 202-217.

Eichenbaum, H., Yonelinas, A. P. and Ranganath, C. (2007). The medial temporal lobe and recognition memory. Annu. Rev. Neurosci. 30, 123-152.

Eichenbaum, H., and Cohen, N. J. (2001). From Conditioning to Conscious Recollection: Memory Systems of the Brain. Oxford: Oxford University Press.

Ekstrom, A. D., Kahana, M. J., Caplan, J. B., Fields, T. A., Isham, E. A., Newman, E. L., and Fried, I. (2003). Cellular networks underlying human spatial navigation. Nature 425, 184-188.

Fenton, A. A., and Muller, R. U. (1998). Place cell discharge is extremely variable during individual passes of the rat through the firing field. Proc. Natl. Acad. Sci. U.S.A. 95, 3182-3187.

Fyhn, M., Hafting, T., Treves, A., Moser, M. B., and Moser, E. I. (2007). 
Hippocampal remapping and grid realignment in entorhinal cortex. Nature 446, 190-194.

Galletti, C., Battaglini, P. P., and Fattori, P. (1995). Eye position influence on the parieto-occipital area $\mathrm{PO}$ (V6) of the macaque monkey. Eur. J. Neurosci. 7, 2486-2501.

Hafting, T., Fyhn, M., Molden, S., Moser, M. B., and Moser, E. I. (2005). Microstructure of a spatial map in the entorhinal cortex. Nature 436, 801-806.

Hannula, D. E., and Greene, A. (2012). The hippocampus re-evaluated in automatic and unconscious expressions of memory: at a tipping point? Front. Hum. Neurosci. 6:80. doi: 10.3389/fnhum.2012.00080

Hartley, T., Bird, C. M., Chan, D., Cipolotti, L., Husain, M., VarghaKhadem, F., and Burgess, N. (2007). The hippocampus is required for short-term topographical memory in humans. Hippocampus 17, 34-48.

Hartley, T., Burgess, N., Lever, C., Cacucci, F., and O'Keefe, J. (2000). Modeling place fields in terms of the cortical inputs to the hippocampus. Hippocampus 10, 369-379.

Hartley, T., Trinkler, I., and Burgess, N. (2004). Geometric determinants of human spatial memory. Cognition 94, 39-75.

Hassabis, D., Kumaran, D., and Maguire, E. A. (2007a). Using imagination to understand the neural basis of episodic memory. J. Neurosci. 27, 14365-14374.

Hassabis, D., Kumaran, D., Vann, S. D., and Maguire, E. A. (2007b). Patients with hippocampal amnesia cannot imagine new experiences. Proc. Natl. Acad. Sci. U.S.A. 104, 1726-1731.

Hayman, R., Verriotis, M. A., Jovalekic, A., Fenton, A. A., and Jeffery, K. J. (2011). Anisotropic encoding of three-dimensional space by place cells and grid cells. Nat. Neurosci. 14, 1182-1188.

Hirsch, R. (1974). The hippocampus and contextual retrieval of information from memory: a theory. Behav. Biol. 12, 421-444.

Holmes, E. A., and Bourne, C. (2008). Inducing and modulating intrusive emotional memories: a review of the trauma film paradigm. Acta Psychol. (Amst.) 127, 553-566.

Huxter, J., Burgess, N., and O'Keefe, J. (2003). Independent rate and temporal coding in hippocampal pyramidal cells. Nature 425, 828-832.

Jacobs, W. J., and Nadel, L. (1985). Stress-induced recovery of fears and phobias. Psychol. Rev. 92, 512-531.

Jeffery, K. J., Anderson, M. I., Hayman, R., and Chakraborty, S. (2004). A proposed architecture for the neural representation of spatial context. Neurosci. Biobehav. Rev. 28, 201-218.

Kim, J. J., and Diamond, D. M. (2002) The stressed hippocampus, synaptic plasticity and lost memories. Nat Rev. Neurosci. 3, 453-462.

Kim, J. J., and Fanselow, M. S. (1992). Modality-specific retrograde amnesia of fear. Science 256, 675-677.

King, J. A., Burgess, N., Hartley, T., Vargha-Khadem, F., and O'Keefe, J. (2002). The human hippocampus and viewpoint dependence in spatial memory. Hippocampus 12, 811-820.

King, J. A., Trinkler, I., Hartley, T., Vargha-Khadem, F., and Burgess, N. (2004). The hippocampal role in spatial memory and the familiarityrecollection distinction: a single case study. Neuropsychology 18, 405-417.

Kinsbourne, M., and Wood, F. (1975). "Short-term memory and the amnesic syndrome," in Short term Memory, eds D. Deutsch, and J. A. Deutsch (New York, NY: Academic), 257-291.

Koenig, J., Linder, A. N., Leutgeb, J. K., and Leutgeb, S. (2011). The spatial periodicity of grid cells is not sustained during reduced theta oscillations. Science 332, 592-595.

LaBar, K. S., Gatenby, J. C., Gore, J. C., LeDoux, J. E., and Phelps, E. A. (1998). Human amygdala activation during conditioned fear acquisition and extinction: a mixed-trial fMRI study. Neuron 20, 937-945.

LeDoux, J. (1996). The Emotional Brain. New York, NY: Simon and Schuster.

Lee, A. C., Buckley, M. J., Pegman, S. J., Spiers, H. J., Scahill, V. R., Gaffan, D., Bussey, T. J., Davies, R. R., Kapur, N., Hodges, J. R., and Graham, K. S. (2005a). Specialisation in the medial temporal lobe for processing of objects and scenes. Hippocampus 15, 782-797.

Lee, A. C., Bussey, T. J., Murray, E. A., Saksida, L. M., Epstein, R. A., Kapur, N., Hodges, J. R., and Graham, K. S. (2005b). Perceptual deficits in amnesia: challenging the medial temporal lobe 'mnemonic' view. Neuropsychologia 43, 1-11.

Lee, A. C., Yeung, L., and Barense, M. D. (2012). The hippocampus and visual perception. Front. Hum. Neurosci. 6:91. doi: 10.3389/fnhum.2012.00091

Leitz, J. R., Morgan, C. J., Bisby, J. A., Rendell, P. G., and Curran, H. V. (2009). Global impairment of prospective memory following acute alcohol. Psychopharmacology (Berl.) 205, 379-387.
Lenck-Santini, P. P., Save, E., and Poucet, B. (2001). Evidence for a relationship between place-cel spatial firing and spatial memory performance. Hippocampus 11, 377-390.

Lever, C., Burgess, N., Cacucci, F., Hartley, T., and O'Keefe, J. (2002) What can the hippocampal representation of environmental geometry tell us about Hebbian learning? Biol. Cybern. 87, 356-372.

Lever, C., Burton, S., Jeewajee, A. O'Keefe, J., and Burgess, N. (2009). Boundary vector cells in the subiculum of the hippocampal formation. J. Neurosci. 29, 9771-9777.

MacDonald, C. J., Lepage, K. Q., Eden, U. T., and Eichenbaum, $\mathrm{H}$ (2011). Hippocampal "time cells" bridge the gap in memory for discontiguous events. Neuron 71 , 737-749.

Manns, J. R., and Eichenbaum, H. (2009). A cognitive map for object memory in the hippocampus. Learn. Mem. 16, 616-624.

Marr, D. (1970). A theory for cerebral cortex. Proc. R. Soc. Lond B Biol. Sci. 176, 161-234.

Marr, D. (1971). Simple memory: a theory for archicortex. Philos. Trans. R. Soc. Lond B Biol. Sci. 262, 23-81.

Matthews, D. B., Simson, P. E., and Best, P. J. (1995). Acute ethanol impairs spatial memory but not stimulus/ response memory in the rat Alcohol. Clin. Exp. Res. 19, 902-909.

Mayes, A., Montaldi, D., and Migo, E. (2007). Associative memory and the medial temporal lobes. Trends Cogn Sci. 11, 126-135.

McClelland, J. L., McNaughton, B. L., and O'Reilly, R. C. (1995). Why there are complementary learning systems in the hippocampus and neocortex: insights from the successes and failures of connectionist models of learning and memory. Psychol. Rev. 102, 419-457.

McNaughton, B. L., Battaglia, F. P., Jensen, O., Moser, E. I., and Moser M. B. (2006). Path integration and the neural basis of the 'cognitive map'. Nat. Rev. Neurosci. 7, 663-678.

Morgan, L., MacEvoy, E., Aguirre, G., and Epstein, R. (2011). Distances between real-world locations are represented in the human hippocampus. J. Neurosci. 31, 1238-1245.

Moscovitch, M. (1995). "Models of consciousness and memory," in The Cognitive Neurosciences, ed M. Gazzaniga (Cambridge, MA: MIT Press), 1341-1356.

Muller, R. U., Bostock, E., Taube, J. S., and Kubie, J. L. (1994). On the directional firing properties of hippocampal place cells. J. Neurosci. 14 , 7235-7251.

Muller, R. U., Kubie, J. L., and Ranck, J. B. Jr. (1987). Spatial firing patterns of hippocampal complex-spike cells in a fixed environment. J. Neurosci. 7, 1935-1950.

Muller, R. U., and Kubie, J. L. (1987) The effects of changes in the environment on the spatial firing of hippocampal complex-spike cells. J. Neurosci. 7, 1951-1968.

Nakazawa, K., Quirk, M. C., Chitwood, R. A., Watanabe, M., Yeckel, M. F., Sun, L. D., Kato, A., Carr, C. A. Johnston, D., Wilson, M. A., and Tonegawa, S. (2002). Requirement for hippocampal CA3 NMDA receptors in associative memory recall. Science 297, 211-218.

Norman, K. A., and O'Reilly, R. C. (2003). Modeling hippocampal and neocortical contributions to recognition memory: a complementarylearning-systems approach. Psychol. Rev. 110, 611-646.

O'Keefe, J. (1976). Place units in the hippocampus of the freely moving rat. Exp. Neurol. 51, 78-109.

O'Keefe, J., and Burgess, N. (1996). Geometric determinants of the place fields of hippocampal neurons. Nature 381, 425-428.

O'Keefe, J., and Nadel, L. (1978). The Hippocampus as a Cognitive Map. Oxford: Oxford University Press.

O'Keefe, J., and Speakman, A. (1987). Single unit activity in the rat hippocampus during a spatial memory task. Exp. Brain Res. 68 $1-27$.

Olsen, R. K., Moses, S. N., Riggs, L., and Ryan, J. D. (2012). The hippocampus supports multiple cognitive processes through relational binding and comparison. Front. Hum. Neurosci. 6:146. doi: 10.3389/fnhum.2012.00146

O'Keefe, J., and Dostrovsky, J. (1971). The hippocampus as a spatial map: preliminary evidence from unit activity in the freely moving rat. Brain Res. 34, 171-175.

Payne, J. D., Jackson, E. D., Hoscheidt, S., Ryan, L., Jacobs, W. J., and Nadel, L. (2007). Stress administered prior to encoding impairs neutral but enhances emotional long-term episodic memories. Learn. Mem. 14, 861-868.

Recce, M., and Harris, K. D. (1996). Memory for places: a navigational model in support of Marr's theory of hippocampal function. Hippocampus 6, 735-748.

Redish, A. D., Rosenzweig, E. S., Bohanick, J. D., McNaughton, B. L., and Barnes, C. A. (2000). Dynamics 
of hippocampal ensemble activity realignment: time versus space. J. Neurosci. 20, 9298-9309.

Rolls, E. T., and Treves, A. (1997). Neural Networks and Brain Function. Oxford: Oxford University Press.

Samsonovich, A., and McNaughton, B. L. (1997). Path integration and cognitive mapping in a continuous attractor network model J. Neurosci. 17, 5900-5920.

Save, E., Paz-Villagran, V., Alexinsky, T., and Poucet, B. (2005). Functional interaction between the associative parietal cortex and hippocampal place cell firing in the rat. Eur. J. Neurosci. 21, 522-530.

Schacter, D. L., Addis, D. R. and Buckner, R. L. (2007). Remembering the past to imagine the future: the prospective brain. Nat. Rev. Neurosci. 8, 657-661.

Shapiro, M. L., Tanila, H., and Eichenbaum, H. (1997). Cues that hippocampal place cells encode: dynamic and hierarchical representation of local and distal stimuli. Hippocampus 7, 624-642.

Smith, M. L., and Milner, B. (1981). The role of the right hippocampus in the recall of spatial location. Neuropsychologia 19, 781-793.

Snyder, L. H., Grieve, K. L., Brotchie, P., and Andersen, R.
A. (1998). Separate body- and world-referenced representations of visual space in parietal cortex. Nature 394, 887-891.

Solstad, T., Boccara, C. N., Kropff, E., Moser, M. B., and Moser, E. I. (2008). Representation of geometric borders in the entorhinal cortex. Science 322, 1865-1868.

Squire, L. R., Stark, C. E., and Clark, R. E. (2004). The medial temporal lobe. Annu. Rev. Neurosci. 27, 279-306.

Squire, L. R., van der Horst, A. S., McDuff, S. G. R., Frascino, J. C., Hopkins, R. O., and Mauldin, K. N. (2010). Role of the hippocampus in remembering the past and imagining the future. Proc. Natl. Acad. Sci. U.S.A. 107, 19044-19048.

Sutherland, R. J., and Rudy, J. W. (1989). Configural association theory: the contribution of the hippocampus to learning, memory, and amnesia. Psychobiology 17, 129-144.

Taube, J. S. (1998). Head direction cells and the neuropsychological basis for a sense of direction. Prog. Neurobiol. $55,225-256$.

Teyler, T. J., and DiScenna, P. (1986). The hippocampal memory indexing theory. Behav. Neurosci. 100, 147-154.
Tolman, E. C. (1948). Cognitive maps in rats and men. Psychol. Rev. 55, 189-208.

Tsivilis, D., Vann, S. D., Denby, C., Roberts, N., Mayes, A. R., Montaldi, D., and Aggleton, J. P. (2008). A disproportionate role for the fornix and mammillary bodies in recall versus recognition memory. Nat. Neurosci. 11, 834-842.

Tulving, E. (1985). Memory and consciousness. Can. Psychol. 26, 1-12.

Vargha-Khadem, F., Gadian, D. G., Watkins, K. E., Connelly, A., van Paesschen, W., and Mishkin, M (1997). Differential effects of early hippocampal pathology on episodic and semantic memory. Science 277 , 376-380.

White, A. M., Elek, T. M., Beltz, T. L., and Best, P. J. (1998). Spatial performance is more sensitive to ethanol than nonspatial performance regardless of cue proximity. Alcohol. Clin. Exp. Res. 22, 2102-2107.

Wills, T., Lever, C., Cacucci, F., Burgess, N., and O'Keefe, J. (2005). Attractor dynamics in the hippocampal representation of the local environment. Science 308 , 873-876.

Zeithamova, D., Schlichting, M. L. and Preston, A. R. (2012). The hippocampus and inferential reasoning: building memories to navigate future decisions. Front. Hum. Neurosci. 6:70. doi: 10.3389/ fnhum.2012.00070

Zhang, K. (1996). Representation of spatial orientation by intrinsic dynamics of the head-direction cell ensemble: a theory. J. Neurosci. 16, 2112-2126.

Conflict of Interest Statement: The authors declare that the research was conducted in the absence of any commercial or financial relationships that could be construed as a potential conflict of interest.

Received: 31 October 2011; accepted: 02 May 2012; published online: 17 May 2012.

Citation: Bird CM, Bisby JA and Burgess $N$ (2012) The hippocampus and spatial constraints on mental imagery. Front. Hum. Neurosci. 6:142. doi: 10.3389/ fnhum.2012.00142

Copyright (c) 2012 Bird, Bisby and Burgess. This is an open-access article distributed under the terms of the Creative Commons Attribution Non Commercial License, which permits non-commercial use, distribution, and reproduction in other forums, provided the original authors and source are credited. 